\title{
Preoperative Positron-Emision Tomography/Computed Tomography Findings in Endometrial Cancer: A Study of 30 Cases
}

\author{
Duygu ALTIN', Dila ZENGIN KASAPOĞLU², Z. Selçuk TUNCER ${ }^{3}$ \\ Ankara, Turkey
}

ABSTRACT

OBJECTIVE: The aim of this study was to find out if preoperative PET/CT could be a substitute for staging surgery and whether SUVmax values correlate with prognostic factors of endometrium cancer.

STUDY DESIGN: 30 patients to whom18-fluorodeoxyglucose PET/CT scan was performed preoperatively were analyzed retrospectively. Standardized uptake value (SUV) was calculated on PET images. PET findings were compared with surgical and pathological findings.

RESULTS: SUVmax of the endometrioid type tumors were significantly higher than non-endometrioid types (p: 0.042). SUVmax significantly differ between histologic grades ( $p: 0.042)$. SUVmax did not differ between patients who had myometrial invasion less or more than $50 \%$ and who had lymphovascular invasion or not. There was not a significant difference between SUVmax and FIGO stages also. Possibility of extra-uterine lesion in PET/CT were significantly higher in late stages (p: 0.004). Sensitivity and specificity of PET/CT for detection of beyond stage 1 disease were $50 \%$ and $67 \%$ respectively and sensitivity and specificity of PET/CT for detection of lymph node metastasis were $40 \%$ and $86 \%$ respectively.

CONCLUSION: PET/CT has moderate sensitivity for pre-operative detection of beyond stage 1 cancers but it has a high specificity and low-moderate sensitivity for detection of lymph node metastasis but has high specificity. Because the positive likelihood ratios are relatively low, PET/CT cannot be a substitute for staging surgery.

Keywords: Endometrial neoplasms, Positron-emission tomography, Diagnostic imaging, Sensitivity and specificity

Gynecol Obstet Reprod Med 2017;23(2):94-99

\section{Introduction}

Endometrial carcinoma is the most common malignancy of female genital tract (1). The lifetime probability of being diagnosed with uterine corpus cancer is $2.8 \%$ (1 in 36) (2). Due to early onset of abnormal bleeding in most of the cases, it is usually diagnosed in early stages $(3,4)$. Currently a total extrafas-

${ }^{1}$ Department of Gynecologic Oncology Ankara University Faculty of Medicine, Ankara

${ }^{2}$ Kings College Hospital, Harris Birthright Research Center London, England

${ }^{3}$ Department of Gynecologic Oncology Hacettepe University Faculty of Medicine, Ankara

Address of Correspondence: Duygu Altin

Department of Gynecologic Oncology, Ankara University Faculty of Medicine, Cebeci, Ankara, Turkey

duyguo@ada.net.tr

Submitted for Publication: $\quad$ 05.01.2017

Accepted for Publication: $\quad 02.03 .2017$

\begin{tabular}{|c|c|}
\hline \multicolumn{2}{|c|}{ Access this article online } \\
\hline \multirow{2}{*}{ 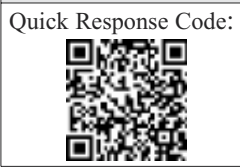 } & Website: www.gorm.com.tr \\
\hline & DOI:10.201613/GORM.2016.657 \\
\hline
\end{tabular}

How to cite this article: Altın D. Zengin Kasapoğlu D. Tuncer ZS. Preoperative Positron-Emision Tomography/Computed Tomography Findings in Endometrial Cancer: A Study of 30 Cases. Gynecol Obstet Reprod Med 2017;23(2):94-9 cial hysterectomy, bilateral salpingo-oophorectomy with pelvic and paraaortic lymph node dissection is the standard staging procedure for endometrial carcinoma (5). The staging system is based on International Federation of Gynecology and Obstetrics criteria after evaluation of pathological findings (FIGO, 2009) (6). The prognosis of endometrial cancer is generally favorable, with $89.6 \%$ five-year survival rate for stage $1 \mathrm{~A}$ disease (7). However, survival figures are much lower if there is lymph node metastasis. Corresponding survival rates were reported to be $57 \%$ and $49 \%$, respectively for pelvic and para-aortic lymph node metastasis in the literature (7).

The role of lymphadenectomy in early stage endometrial cancer is controversial since the therapeutic value of the procedure remains unclear (8). The incidence of lymph node metastasis in early disease is reported to be relatively low ranging from $3 \%$ to $20 \%$ (9). Furthermore, lymphadenectomy procedure has significant immediate or delayed risks including bleeding and lymphocele formation (10). If we were able to determine preoperatively whether there is metastasis to lymph nodes or not, then we would prevent unnecessary lymphadenectomy and potential complications.

Positron emission tomography (PET) uses 18fluoro-2-deoxyglucose (FDG) to detect increase of glucose uptake in malignant cells (11). FDG is taken up by the cells with glucose transporters and phosphorylated by hexokinase. FDG-6- phos- 
phate does not go further metabolized by glycolytic enzymes and accumulate in cells. FDG especially accumulates in cells of brain, heart or tumor tissue, which have enhanced glucose metabolism (12). This makes PET a functional imaging method and integration of computed tomography (CT) to it results in further anatomic information. Thus, both metabolic and anatomic evaluation of lesions can be performed with $\mathrm{PET} / \mathrm{CT}$ and results are expected to be superior to magnetic resonance imaging (MRI) or CT alone, which detects only structural changes (13).

Imaging techniques including CT and MRI were used to assess the extent of disease preoperatively in several studies, however, their role was found to be limited when compared to surgical staging (14). PET/CT, as a relatively new imaging technique, was also used in endometrial cancer with the hope of more rationale management (15). There have been several studies regarding the use of PET/CT in detection of lymph node metastasis, however, limited data about the correlation of preoperative PET/CT findings with surgical pathological findings (16-18). A study among 30 consecutive cases that were managed surgically in a single institution was done with the purpose of evaluation diagnostic accuracy of PET/CT in detecting nodal and extra-uterine metastases as well as other pathological parameters.

\section{Material and Method}

\section{Patients}

This study includes retrospective review of 30 patients with endometrial cancer who underwent a PET/CT scan prior to surgical staging procedure. All the patients had total abdominal hysterectomy, bilateral salpingo-oophorectomy, omentectomy, pelvic and para-aortic lymphadenectomy and peritoneal cytology at Hacettepe University Hospital between December 2011 and December 2014. The institution's review board approved this retrospective study. Patients' imaging studies, histological, clinical and laboratory data were collected from institution's computerized database.

\section{Procedure}

FIGO stage, lymphovascular space invasion (LVSI), tumor size, histological grade, depth of myometrial invasion, cervical and extra-uterine metastasis and lymph node metastasis were obtained from the operation and pathology reports. SUVmax value of the uterine lesion, size of the lesion, localization of extra-uterine pathological uptakes and their SUVmax values were the parameters studied for PET/CT.

\section{Statistical Analysis}

Descriptive data were presented as percentages or means (SDs). In order to compare variables, Mann-Whitney U test, Kruskal Wallis $\mathrm{H}$ test, One-Way Anova test and Chi-square test were used. All statistical tests were two-sided and significance was set at $\mathrm{p}<0.05$. SPSS software for Windows (version 17.0; IBM SPSS, Somers, NY) was used for all analysis.
To find out sensitivity and specificity of PET/CT for detection of distant spread and lymph node metastasis a crosstable was made. The parameters counted as per place of metastasis or per node. By the definition, a true positive lesion was a lesion detected on PET/CT images and found to be positive for tumor tissue (metastasis) at histologic analysis. A false positive lesion was a lesion seen on PET/CT images but found to be negative for tumor tissue (metastasis) at histologic analysis. A true negative lesion was indicated when no lesion was seen on PET/CT images and result of the histologic analysis was negative for tumor tissue. A false negative lesion was a lesion that was missed at PET/CT but was found to be positive for neoplastic tissue at histologic analysis. Sensitivity, specificity, positive predictive value (PPV) and negative predictive value (NPV) of PET/CT imaging for cervix plus extrauterine metastasis, and lymph node metastases were calculated separately. 95\% confidence intervals were determined.

\section{Results}

The mean age of the patients was $59.8 \pm 6.6$ years. PET/CT was performed within $3.67 \pm 3.93$ days. Patient characteristics are presented in Table 1.

Table 1: Patient characteristics and histological findings

\begin{tabular}{ll}
\hline Parameter & Number (\%) \\
\hline Age, mean & $59.8 \pm 6.6$ years \\
Tumor type & \\
$\quad$ Endometrioid & $23(76.7)$ \\
Non-endometrioid & $7(23.3)$ \\
FIGO Stage & \\
1 & $18(60)$ \\
2 & $4(13.3)$ \\
3 & $5(16.7)$ \\
4 & $3(10)$ \\
Grade & \\
1 & $16(57.1)$ \\
2 & $5(17.9)$ \\
3 & $7(25)$ \\
Myometrial invasion & \\
$<\% 50$ & $17(56.7)$ \\
$>\% 50$ & $13(43.3)$ \\
Lymphovascular space invasion & $6(21.4)$ \\
Positive & $22(78.6)$ \\
Negative & \\
\hline
\end{tabular}

Among the 30 patients studied, 23 (76.7\%) had endometrioid type adenocarcinoma, $3(10 \%)$ had clear cell carcinoma, two had (6.7\%) serous carcinoma and 2 (6.7\%) had undifferentiated carcinoma. Uterine SUVmax of the endometrioid type was significantly higher than the uterine SUVmax of the non-endometrioid type [Endometrioid: 16.6 (5.7-24.7); non-endometrioid 9.2 (3.2-19.0); $Z=-2.01$, $\mathrm{p}=0.042]$ (Figure 1). 


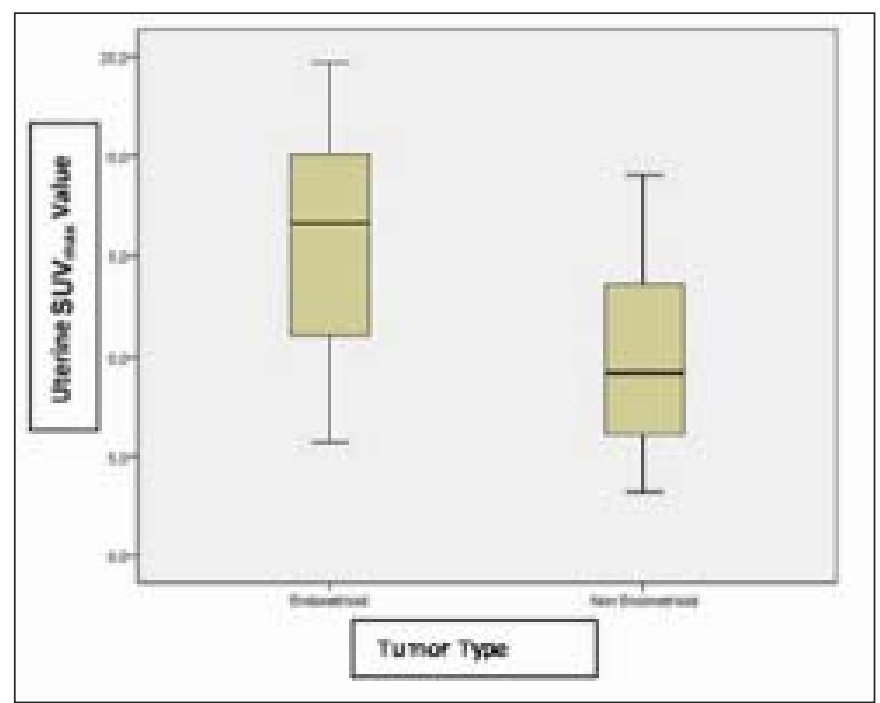

Figure 1: Correlation of uterine SUVmax values between endometrioid and non-endometrioid types

Of the patients, $16(57.1 \%)$ had grade 1, 5 (17.9\%) had grade 2 and 7 (25\%) had grade 3 tumor. Grade was not defined in two patients. All non-endometrioid types were counted as grade 3 because of the tumor's nature. SUVmax was significantly different between histologic grades $(F=3.61, p=0.042)$. In the post-hoc analyses, it was found that SUVmax of grade $2(X=19.14 \pm 2.35)$ and grade $3(X=10.13 \pm 5.59)$ lesions were statistically significant (Figure 2).

The SUVmax value of the cases with myometrial invasion less than $1 / 2$ (n: 17, mean SUVmax: 14.8) was lower than the cases with myometrial invasion more than $1 / 2$ (n:13, mean SUVmax: 17.1), but this difference was not statistically significant $(Z=-0.27, p=0.78)$. It was also not significant for the endometrioid subtype $(\mathrm{Z}=-1.0, \mathrm{p}=0.32)$. Lymphovascular space invasion was not defined in two patients. Among the 28 patients, no significant difference was observed between
SUVmax value of patients who had LVSI ( $n=6$, SUVmax $=17.5)$ and who did not $(n=22$, SUVmax $=13.5)(Z=-0.028$, $\mathrm{p}=0.978)$. The SUVmax value didn't change as the tumor size enlarges $(r=0.074, \mathrm{P}=0.70)$.

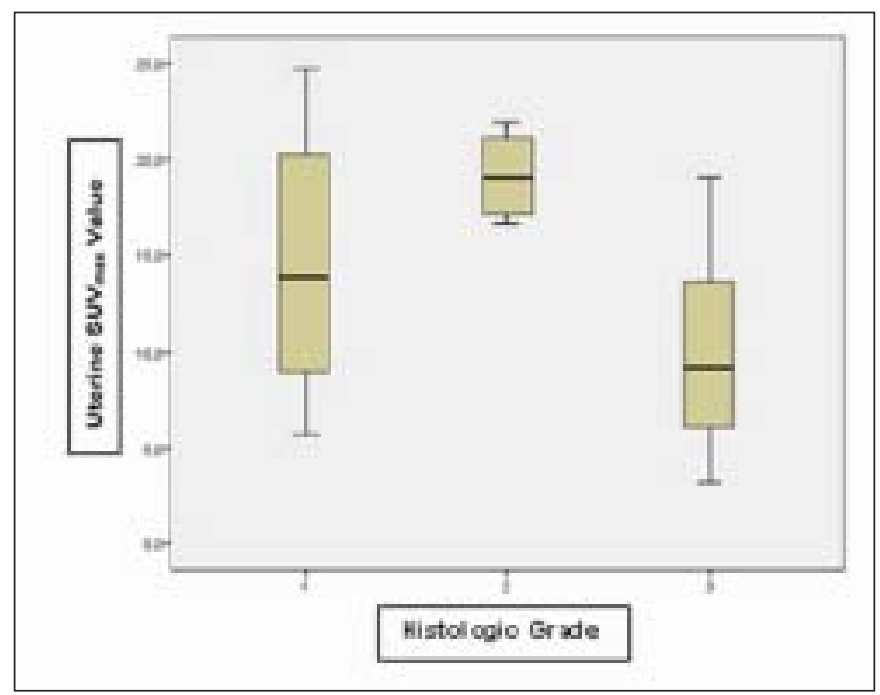

Figure 2: Correlation of uterine SUVmax values between histologic grades

The correlation of SUVmax of the uterine tumor and FIGO stage was presented in Table 2. There was no significant difference among different stages $(\mathrm{X}=2.22, \mathrm{p}=0.528)$. There was also no significant difference when stage 1 and 2 are considered as early stage (mean SUVmax value: 14.1 ) and stage 3 and 4 are considered as late stage (mean SUVmax value: 15.2 ) $(\mathrm{Z}=-0.49, \mathrm{p}=0.62)$.

We analyzed if there was a relationship between the FIGO stages and existence of extra-uterine lesions in PET/CT. The stages were defined as early (stage 1 and 2) and late (stage 3 and 4) stages. The possibilities of extra-uterine lesion in $\mathrm{PET} / \mathrm{CT}$ were significantly higher in late stages $(\mathrm{X}=8.52$, $\mathrm{p}=0.004$ ) (Table 3).

Table 2: Correlation of uterine sUVmax values between FIGO stages (Kruskal Wallis Test)

\begin{tabular}{|l|l|l|}
\hline FIGO Stage (number of cases) & Uterine SUVmax values [median (min-max)] & \\
\hline Stage $1(18)$ & $12.0(3.4-24.7)$ & $\mathrm{x}=2.22$, \\
\hline Stage $2(4)$ & $20.5(6.6-22.0)$ & $\mathrm{p}=0.528$ \\
\hline Stage $3(5)$ & $15.6(5.6-17.9)$ & \\
\hline Stage $4(3)$ & $14.8(12.5-19.0)$ & \\
\hline
\end{tabular}

Table 3: Correlation between FIGO stages and existence of extra-uterine lesions in PET/CT (Chi-Square Test)

\begin{tabular}{|l|l|l|l|l|}
\hline & Early Stage & Late Stage & Total & \\
\hline PET/CT EUD + & $4(\% 13.3)$ & $2(\% 6.7)$ & $6(\% 20.0)$ & \\
\hline PET/CT EUD - & $18(\% 60.0)$ & $6(\% 20.0)$ & $24(\% 80.0)$ & $x=8.52$, \\
\hline Total & $22(\% 73.3)$ & $8(\% 26.7)$ & $30(\% 100)$ & \\
\hline
\end{tabular}


The sensitivity, specificity, positive predictive value (PPV), negative predictive value (NPV) of PET/CT for detection of cervix plus extra-uterine lesions (stage 2 or more) were $50 \%, 67 \%, 47 \%$, and $69 \%$, respectively (Table 4 ). The sensitivity, specificity, PPV and NPV of PET/CT for detection of lymph node metastasis were $40 \%, 86 \%, 33 \%$ and $89 \%$, respectively (Table 5).

\section{Discussion}

A non-invasive preoperative technique that stages endometrial cancer would be beneficial for patients who will undergo surgery. If we would accurately know the stage and place of metastasis preoperatively, unnecessary lymph node dissections and more hysterectomies that are radical would be avoided. CT, MRI and ultrasonography were used for this purpose, but the accuracy is low (14). The aim of this study was to compare the effectiveness of preoperative PET/CT with surgery and histologic results.

An interesting finding of this study was SUVmax of endometrioid subtype was higher than non-endometrioid types. There are findings about the difference between metabolisms of endometrioid and non-endometrioid type endometrial cancers $(19,20)$. Authors reported higher SUVmax values in nonendometrioid types (21). Our finding differs from other studies suggesting that there may be other factors influencing SUVmax.

In previous studies, some authors found SUVmax correlates with histologic grade $(22,23)$ and some did not $(24,25)$. In our study, we found that grade 2 tumors had statistically not important but higher SUVmax values than grade 1 tumors and statistically significantly higher SUVmax values than grade 3 tumors. Grade 3 tumors had relatively lower SUVmax values because non-endometrioid types, which count as grade 3 tumors, had lower SUVmax values. The range of grade 1 tumors' SUVmax values (5.7-24-7) were higher than grade 2 tumors' (16.6-21.9) which may had affected our results.

Our results also differ from the literature since there was no statistically significant difference between SUVmax and myometrial invasion depth nor existence of lymphovascular space invasion and the stage $(18,22,26)$. LVSI is not a part of staging but existence of LVSI is a bad prognostic factor (27). Existence of LVSI is especially important for giving adjuvant therapy decision in patients who had not undergone lymph node dissection (28). This finding is interesting because the tumor with deep myometrial invasion or LVSI has more aggressive nature. As we just had 30 patients, larger sample sized studies are needed.

The detection of beyond stage 1 disease for PET/CT was not explored in previous studies; instead, detection of lymph node or metastasis was searched separately. It is important to know or predict the stage of disease before the operation to perform full staging surgery. In previous studies, it was found that PET/CT has moderate sensitivity and high specificity for detecting nodal metastasis (29-31) and high sensitivity and specificy for detecting metastatic disease (32). Authors concluded that PET/CT is not a substitute for lymphadenectomy. In recent studies, PET/CT was found more effective in detecting lymph node metastasis in high-risk patients and it can be utilized in only high-risk patients with comorbidities $(33,34)$.

Table 4: Sensitivity and specificity of PET/CT (Index Method) for detection of cervix + extra-uterine disease according to surgical staging (standard method)

\begin{tabular}{|c|c|c|c|c|}
\hline & $\begin{array}{l}\text { Standard } \\
\text { Method + }\end{array}$ & $\begin{array}{l}\text { Standard } \\
\text { Method - }\end{array}$ & Total & Sensitivity, specificity, positive and negative likelihood ratio of PET/CT \\
\hline Index Method + & 7 & 8 & 15 & \multirow{3}{*}{$\begin{array}{l}\text { Sensitivity: } 0.500 \text { (\%95Cl: } 0.268-0.732) \\
\text { Specificity: } 0.667 \text { (\%95 Cl: } 0.467-0.820) \\
\text { Positive likelihood ratio: } 1.500 \text { (\%95 Cl: } 0.694-3.243) \\
\text { Negative likelihood ratio: } 0.750 \text { (\%95 Cl: } 0.414-1.360)\end{array}$} \\
\hline Index Method - & 7 & 6 & 13 & \\
\hline Total & 14 & 24 & 18 & \\
\hline
\end{tabular}

The parameters counted as per place of metastasis and per lymph node number, not a region

Table 5: Sensitivity and specificity of PET/CT (Index Method) for detection of lymph node metastasis according to surgical staging (standard method)

\begin{tabular}{|c|c|c|c|c|}
\hline & $\begin{array}{l}\text { Standard } \\
\text { Method + }\end{array}$ & $\begin{array}{l}\text { Standard } \\
\text { Method - }\end{array}$ & Total & Sensitivity, specificity, positive and negative likelihood ratio of PET/CT \\
\hline Index Method + & 2 & 4 & 6 & \multirow{3}{*}{$\begin{array}{l}\text { Sensitivity: } 0.400 \text { (\%95Cl: } 0.117-0.769) \\
\text { Specificity: } 0.857 \text { (\%95 Cl: } 0.685-0.943) \\
\text { Positive likelihood ratio: } 2.800 \text { (\%95 Cl: } 0.687-11.418) \\
\text { Negative likelihood ratio: } 0.700 \text { (\%95 Cl: } 0.500-31.981)\end{array}$} \\
\hline Index Method - & 3 & 24 & 27 & \\
\hline Total & 5 & 28 & 13 & \\
\hline
\end{tabular}


We found that PET/CT has moderate sensitivity for preoperative detection of beyond stage 1 cancers but it has a high specificity and low-moderate sensitivity for detection of lymph node metastasis but has high specificity. The positive likelihood ratio is 1.5 for beyond stage 2 disease and 2.8 for lymph node metastasis. This means if the result is positive in PET/CT both for beyond stage 1 disease and lymph node metastasis, it is reasonable to assume that there is really extrauterine disease exists. Hence, the positive likelihood ratios are relatively low this hypothesis is weakened. If there is no lesion detected on PET/CT, it is rational not to assume there is no metastasis. Lymphadenectomy is still needed even the $\mathrm{PET} / \mathrm{CT}$ is negative both for cervical + extra-uterine disease and lymph nodes.

Our study had some limitations. First, the number of patients involved was relatively small. Second, this was a retrospective study. Third PET/CT was not performed to every patient with endometrial cancer. The use of PET/CT in only selected patients might have caused bias. Therefore, prospective studies with large number of patients are needed to find out if PET/CT can be a substitute for staging surgery.

\section{References}

1. Torre LA, Bray F, Siegel RL, Ferlay J, Lortet-Tieulent J, Jemal A. Global cancer statistics, 2012. CA Cancer J Clin 2015 Mar; 65(2):87-108.

2. Siegel RL, Miller KD, Jemal A. Cancer statistics, 2016. CA Cancer J Clin 2016 Jan-Feb; 66(1):7-30.

3. American College of Obstetricians and Gynecologists. ACOG practice bulletin, clinical management guidelines for obstetrician-gynecologists, number 65, August 2005: management of endometrial cancer. Obstet Gynecol 2005 Aug;106(2):413-25.

4. Pakish JB, Lu KH, Sun CC, Burzawa JK, Greisinger A, Smith FA, et al. Endometrial cancer associated symptoms: a case-control study. J Womans Health (Larchmt) 2016 Nov; 25(11):1187-1192.

5. Lewin SN. Revised FIGO staging system for endometrial cancer. Clin Obstet Gynecol 2011 Jun; 54(2):215-8.

6. Pecorelli S. Revised FIGO staging for carcinoma of the vulva, cervix and endometrium. Int J Gynaecol Obstet 2009 May; 105(2):103-4.

7. Lewin SN, Herzog TJ, Barrena Medel NI, Deutsch I, Burke WM, Sun X, et al. Comparative performance of the 2009 international federation of gynecology and obstetrics' staging system for uterine corpus cancer. Obstet Gynecol 2010 Nov; 116(5):1141-9.

8. Chan JK, Kapp DS. Role of complete lymphadenectomy in endometrioid uterine cancer. Lancet Oncol 2007 Sep; 8(9):831-41.

9. The writing committee on behalf of the ASTEC study group. Efficacy of systematic pelvic lymphadenectomy in endometrial cancer (MRS ASTEC trial): a randomized study. Lancet 2009 Jan 10; 373(9658):125-36.

10. Zikan M, Fischerova D, Pınkakova I, Slama J, Weinberger $\mathrm{V}$, Dusek L, et al. A prospective study examining the incidence of asymptomatic and symptomatic lymphoceles following lymphadenectomy in patients with gynecological cancer. Gynecol Oncol 2015 May; 137(2):291-8.

11. Kinahan PE, Townsend DW, Beyer T, Sashin D. Attenuation correction for a combined 3D PET/CT scanner. Med Phys 1998 Oct; 25(10):2046-53.

12. Horowitz NS, Dehdasti F, Herzog TJ, Rader JS, Powell MA, Gibb RK, et al. Prospective evaluation of FDG-PET for detecting pelvic and para-aortic lymph node metastasis in uterine corpus cancer. Gynecol Oncol 2004 Dec; 95(3):546-51.

13. Beyer T, Townsend DW, Brun T, Kinahan PE, Charron M, Roddy R, et al. A combined PET/CT scanner for clinical oncology. J Nucl Med 2000 Aug; 41(8):1369-79.

14. Kinkel K, Kaji Y, Yu KK, Segal MR, Lu Y, Powell CB, et al. Radiologic staging in patients with endometrial cancer: a meta-analysis. Radiology 1999 Sep; 212(3):711-8.

15. Nogami Y, Iida M, Banno K, Kisu I, Adachi M, Nakamura $\mathrm{K}$, et al. Application of FDG-PET in cervical cancer and endometrial cancer: utility and future prospects. Anticancer Res 2014 Feb; 34(2):585-92.

16. Haldorsen IS, Salvesen HB. What is the best preoperative imaging for endometrial cancer? Curr Oncol Rep 2016 Apr; 18(4):25.

17. Walentowicz-Sadlecka, Sadlecki P, Malecki B, Walentowicz P, Marszalek A, Domaracki P, et al. SUVmax measured by $18 \mathrm{~F}$ FDG PET/CT in the primary tumor in relation to clinical and pathological features of endometrial cancer. Ginekol Pol 2013 Sep; 84(9):748-53.

18. Antonsen SL, Loft A, Fisker R, Nielsen AL, Andersen ES, Hogdall E, et al. SUVmax of 18FDGPET/CT as a predictor of high-risk endometrial cancer patients. Gynecol Oncol 2013 May;129(2):298-303.

19. Hayes MP, Douglas W, Ellenson LH. Molecular alterations of EGFR and PIK3CA in uterine serous carcinoma. Gynecol Oncol 2009 Jun;113(3):370-3.

20. Shoji K, Oda K, Nakagawa S, Hosokawa S, Nagae G, Uehara $\mathrm{Y}$, et al. The oncogenic mutation in the pleckstrin homology domain of AKT1 in endometrial carcinomas. Br J Cancer 2009 Jul 7;101(1):145-8.

21. Lee HJ, Ahn BC, Hong CM, Song BI, Kim HW, Kang S, et al. Preoperative risk stratification using (18)F-FDG $\mathrm{PET} / \mathrm{CT}$ in women with endometrial cancer. Nuklearmedizin 2011;50(5):204-13.

22. Nakamura K, Kodama J, Okumura Y, Hongo A, Kawazawa S, Hiramatsu Y. The Suv-max of 18FFDG PET correlates with histological grade in endometrial cancer. Int J Gynecol Cancer 2010 Jan; 20(1):110-5. 
23. Walentowicz-Sadlecka M, Malkowski B, Walentowicz P, Sadlecki P, Marszalek A, Pietrzak T, et al. The preoperative maximum standardized uptake value measured by 18F-FDG PET/CT as an independent prognostic factor of overall survival in endometrial cancer patients. Biomed Res Int 2014; 2014:234813.

24. Torizuka T, Nakamura F, Takekuma M, Kanno T, Ogusu T, Yoshikawa E, et al. FDG PET for the assessment of myometrial infiltration in clinical stage I uterine corpus cancer. Nucl Med Commun 2006 Jun; 27(6):481-7.

25. Kitajima K, Suenaga Y, Ueno Y, Maeda T, Ebina Y, Yamada $\mathrm{H}$, et al. Preoperative risk stratification using metabolic parameters of (18)F-FDG PET/CT in patients with endometrial cancer. Ear J Null Med Mol Imaging 2015 Jul;42(8):1268-75.

26. Kitajima K, Kita M, Suzuki K, Senda M, Nakamoto Y, Sugimura K. Prognostic significance of SUVmax (maximum standardized uptake value) measured by $18 \mathrm{~F}$ FDG $\mathrm{PET} / \mathrm{CT}$ in endometrial cancer. Eur J Nucl Med Mol Imaging 2012 May; 39(5):840-5.

27. Güven D, Bakay K, Koçak İ, Üstün C. Endometrial Cancer-Evaluation of Diagnostics Treatment and Prognosis in 150 Patients. Gynecol Obstet Reprod Med 2012;18(1):21-5.

28. Mariani A, Webb MJ, Keeney GL, Lesnick TG, Podratz KC. Surgical stage I endometrial cancer: predictors of distant failure and death. Gynecol Oncol 2002 Dec; $87(3): 274-80$

29. Chang MC, Chen JH, Liang JA, Yang KT, Cheng KY, Kao CH. 18F-FDG PET or PET/CT for detection of metastatic lymph nodes in patients with endometrial cancer: a systematic review and meta-analysis. Eur J Radiol 2012 Nov; 81(11):3511-7.

30. Kitajima K, Murakami K, Yamasaki E, Fukasawa I, Inaba N, Kaji Y, et al. Accuracy of 18F-FDG PET/CT in detecting pelvic and paraaortic lymph node metastasis in patients with endometrial cancer. AJR Am J Roentgenol 2008 Jun; 190(6):1652-8.

31. Chung HH, Cheon GJ, Kim HS, Kim JW, Park NH, Song YS. Preoperative PET/CT standardized FDG uptake values of pelvic lymph nodes as a significant prognostic factor in patients with endometrial cancer. Eur J Nucl Med Mol Imaging 2014 Sep; 41(9):1793-9.

32. Suga T, Nakamoto Y, Saga T, Higashi T, Hamanaka Y, Tatsumi M, et al. Clinical value of FDG-PET for preoperative evaluation of endometrial cancer. Ann Nucl Med 2011 May; 25(4):269-75.

33. Konuralp Atakul B, Taşkın S, Soydal Ç, Şükür YE, Kahraman A, Koyuncu K, et al. Preoperative 18FFluorodeoxyglucose Positron Emission Tomography/CT in Prediction of Uterine Risk Factors and Lymph Node Metastasis: An Analysis of 111 Endometrioid Endometrial Cancer Patients. Gynecol Obstet Invest 2016 Oct 22 DOI: $10.1159 / 000452100$

34. Atri M, Zhang Z, Dehdashti F, Lee SI, Marques H, Ali S, et al. Utility of PET/CT to evaluate Retroperitoneal Lymph Node Metastasis in High-Risk Endometrial Cancer: Results of ACRIN 6671/GOG 0233 Trial. Radiology 2017 Jan 3:160200 DOI:10.1148/radiol. 2016160200 